\title{
LIDAR-DERIVED ELEVATION DATA FOR SITKA, ALASKA
}

Ronald P. Daanen, Gabriel J. Wolken, and Andrew M. Herbst Raw Data File 2020-13

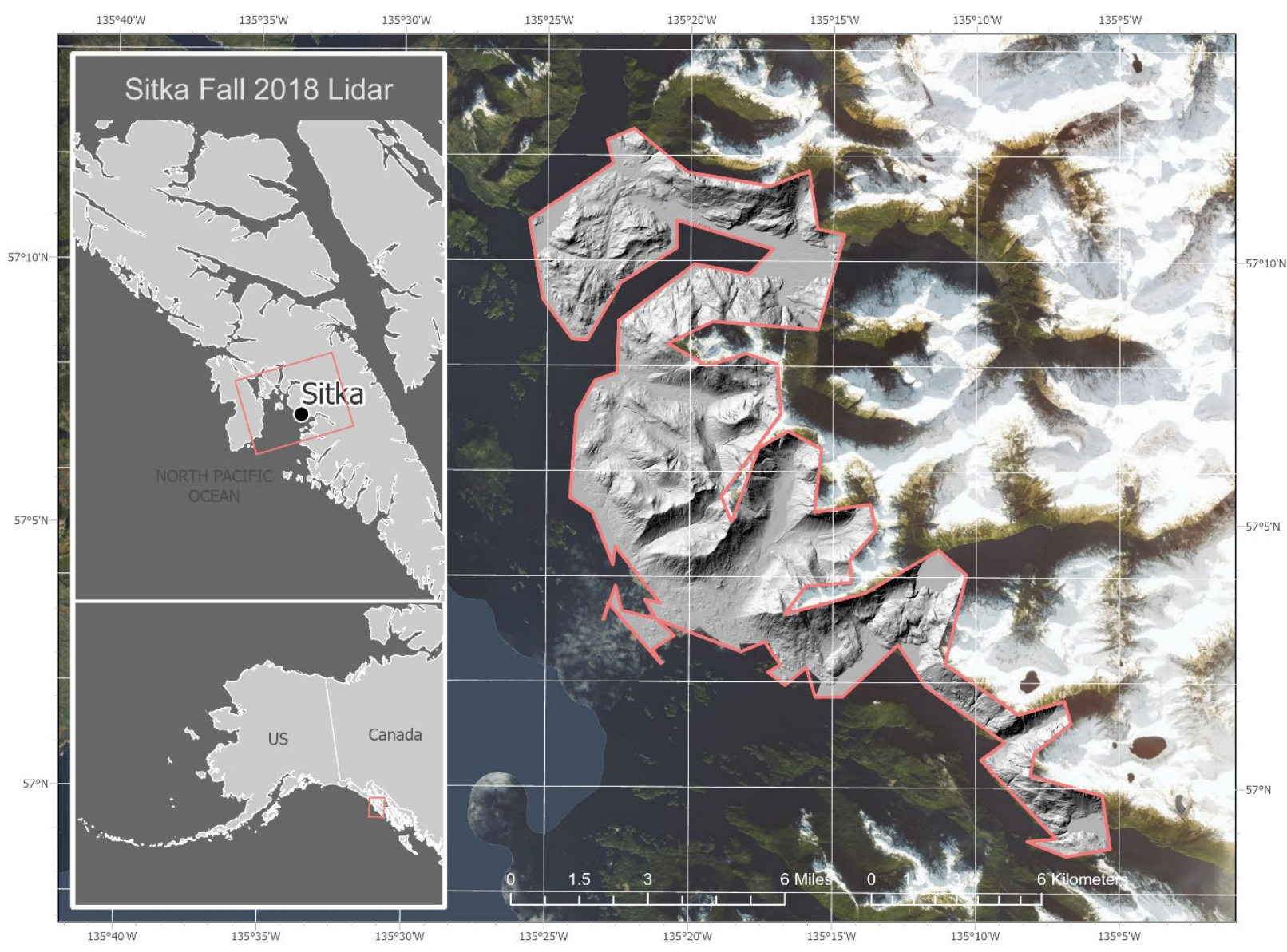

Location map of survey area with ortho-image base layer.

This report has not been reviewed for technical content or for conformity to the editorial standards of DGGS.

2020

STATE OF ALASKA

DEPARTMENT OF NATURAL RESOURCES

DIVISION OF GEOLOGICAL \& GEOPHYSICAL SURVEYS
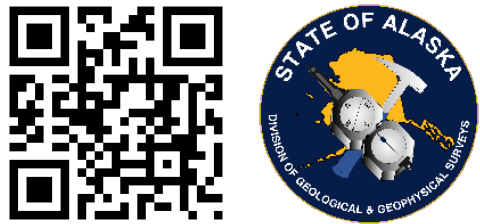
STATE OF ALASKA

Mike Dunleavy, Governor

DEPARTMENT OF NATURAL RESOURCES

Corri A. Feige, Commissioner

\section{DIVISION OF GEOLOGICAL \& GEOPHYSICAL SURVEYS}

Steve Masterman, State Geologist \& Director

Publications produced by the Division of Geological \& Geophysical Surveys are available to download from the DGGS website (dggs.alaska.gov). Publications on hard-copy or digital media can be examined or purchased in the Fairbanks office:

\section{Alaska Division of Geological \& Geophysical Surveys (DGGS)}

3354 College Road | Fairbanks, Alaska 99709-3707

Phone: 907.451 .5010 | Fax 907.451.5050

dggspubs@alaska.gov | dggs.alaska.gov

DGGS publications are also available at:

Alaska State Library, Historical

Collections \& Talking Book Center

395 Whittier Street

Juneau, Alaska 99801

Alaska Resource Library and

Information Services (ARLIS)

3150 C Street, Suite 100

Anchorage, Alaska 99503

\section{Suggested citation:}

Daanen, R.P, Wolken, G.J., Herbst A.M, 2020, Lidar-derived elevation data for Sitka, Alaska: Alaska Division of Geological \& Geophysical

Surveys Raw Data File 2020-13, 11 p. http://doi.org/10.14509/30531
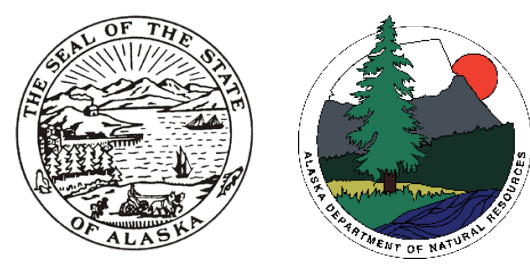


\section{LIDAR-DERIVED ELEVATION DATA FOR SITKA, ALASKA}

Ronald P. Daanen', Gabriel J. Wolken', and Andrew M. Herbst ${ }^{1}$

\section{ABSTRACT}

The State of Alaska Division of Geological \& Geophysical Surveys (DGGS) produced airborne lidar-derived elevation data for Sitka, Alaska. This data collection is being released as a Raw Data File with an open end-user license. All files can be downloaded, free of charge, from the DGGS website at https://doi.org/10.14509/30531.

\section{INTRODUCTION}

These data were produced to assess bare earth slope conditions as part of an overarching, multi-hazard risk analysis for the study area, coordinated through the Federal Emergency Management Agency (FEMA) Cooperating Technical Partners (CTP) program. The project was initiated in response to a tragic debris flow incident that took three Alaskans' lives in 2015.

\section{LIST OF DELIVERABLES}

Classified Points

Canopy Height Model (CHM)

Digital Terrain Model (DTM)

Digital Surface Model (DSM)

Lidar Intensity Image

Metadata

\section{MISSION PLAN}

\section{Airborne Survey Details}

This dataset includes point cloud data, a digital terrain model (DTM), digital surface model (DSM), canopy height model (CHM), and an intensity image covering Sitka, Alaska, and the surrounding area (approximately $60 \mathrm{mi}^{2}\left[155 \mathrm{~km}^{2}\right]$ ). This survey was conducted with a Riegl VUX1-LR lidar scanner with an integrated GNSS and Northrop Grumman IMU system. The integration was designed by Phoenix LiDAR systems. This survey was flown with a pulse rate between 200,000-400,000 pulses/second and at a scan rate between 80 and 150 revolutions/second. This survey was flown with an average elevation of $400 \mathrm{~m}$ above ground level and a ground speed of approximately $40 \mathrm{~m} / \mathrm{s}$ with a fixed-wing Cessna 185 aircraft. The scan angle was set from 55 to 305 degrees, centered normal to the aircraft's bottom.

${ }^{1}$ Alaska Division of Geological \& Geophysical Surveys, 3354 College Road, Fairbanks, Alaska 99709 


\section{Weather Conditions and Flight Times}

Airborne and ground surveys occurred between the $27^{\text {th }}$ and $30^{\text {th }}$ of April, 2018. Weather was partially cloudy throughout the survey, at times obscuring higher altitude topography in the study area. These conditions limited data coverage to areas below 980 meters elevation.

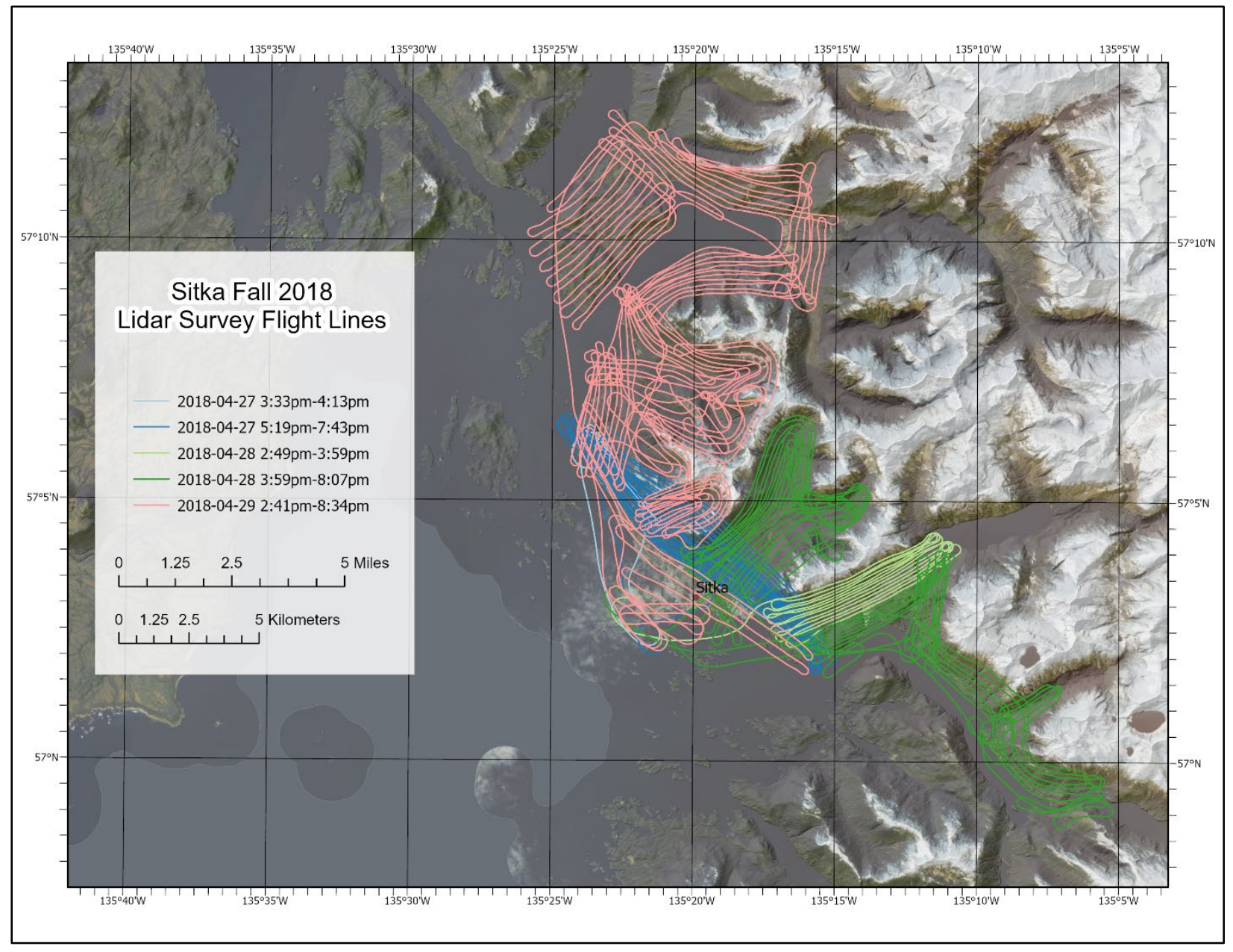

Figure 1. Project flightlines.

\section{PROCESSING REPORT}

\section{Lidar Dataset Processing}

SDCImport software was used to apply range thresholding, reflectance thresholding, and missed-time-around (MTA) disambiguation for preliminary point cloud noise filtering. In-flight IMU and GNSS data were coupled using Inertial Explorer software to produce the trajectory data. Spatial Explorer software was used to couple the trajectory data with the point cloud.

The point cloud data were calibrated using tielines for roll, pitch, and yaw of the aircraft during the survey. This process was first run using all points, then on a per-flightline basis for 
additional accuracy. Interswath fluctuations in ground points were identified using overlapping tielines to further calibrate the data. The point cloud was classified in accordance with American Society for Photogrammetry and Remote Sensing (ASPRS) guidelines using project-tailored macros. Misclassified points were manually reclassified in post-processing QA/QC. The point cloud was converted from ellipsoidal to orthometric heights using geoid $12 \mathrm{~B}$, then uniformly adjusted to maintain a mean offset of 0 with collected ground control. Calibration, classification, and height adjustments were all executed in TerraSolid.

Derived products were processed in ArcMap. DTM and DSM were produced using point triangulation with nearest-neighbor interpolation. The DTM was derived from all returns for ground classified points, while the DSM used first returns for all non-noise classes. The CHM was created by subtracting DTM height values from DSM height values. A lidar intensity image was created from first returns of all classes using mean binning.

\section{Lidar Intensity Image}

The lidar intensity image includes a range of values between $30.6 \mathrm{k}$ and $33.6 \mathrm{k}$ and describes the relative amplitude of received signals in the point cloud. Lidar intensity is largely a function of scanned object reflectance in relation to the signal frequency and is not necessarily consistent between separate scans. The intensity image is a single-band, 32-bit float GeoTIFF file with a ground sample distance of 0.5 meters. No Data value is set to $-3.40282306074 \mathrm{e}+038$ (32-bit, floating-point minimum).

\section{Canopy Height Model}

The CHM displays vegetation heights as the difference between DSM and DTM heights. The CHM is a single-band, 32-bit float GeoTIFF file with a ground sample distance of 0.5 meters. No Data value is set to $-3.40282306074 \mathrm{e}+038$. Note: there are some negative values in the CHM, present in low confidence areas of the DSM and DTM.

\section{Digital Terrain Model}

The DTM represents elevations of the ground surface by penetrating or flattening any vegetation, bridges, buildings, etc. The DTM is a single-band, 32-bit float GeoTIFF file with a ground sample distance of 0.5 meters. No Data value is set to $-3.40282306074 \mathrm{e}+038$.

\section{Digital Surface Model}

The DSM represents human-observable surface elevations, which include vegetation, buildings, etc. The DSM is a single band, 32-bit GeoTIFF file with a ground sample distance of 0.5 meters. No Data value is set to $-3.40282306074 \mathrm{e}+038$.

\section{Classified Point Cloud}

Classified point cloud data is provided in this collection in compressed laz format. An average point spacing of 0.15 meters was calculated from all returns and all non-noise classes. 


\section{SURVEY REPORT}

\section{Ground Survey Details}

Trimble R8 and R9 RTK GPS systems were used to collect 195 survey points, 100 of which were used as control points and 95 of which were used as checkpoints. Points were gathered by vehicle along the road system and by boat along the coastline. Points were adjusted for accuracy according to OPUS corrections in Trimble Business Center.

\section{Coordinate system and Datum}

All data were processed and delivered in NAD83 UTM8N and vertical datum NAVD88, GEOID12B.

\section{Vertical Accuracy}

One hundred ground control points were used to determine a $-0.015 \mathrm{~m}$ average offset from the point cloud (appendix 1), which was corrected with a uniform z-transformation of the lidar data. Point cloud accuracy was measured using 95 checkpoints to determine a root-mean-square error of $0.063 \mathrm{~m}$ for the project (appendix 2). Relative accuracy for this dataset has been evaluated as the interswath consistency, measured by comparing tie line points within swath overlap areas. The interswath root-mean-square error was calculated to be $0.008 \mathrm{~m}$.

\section{ACKNOWLEDGMENTS}

Funding was provided by the State of Alaska and by National Park Service awards \#P17AC00903 and award \#P15AC01879. 


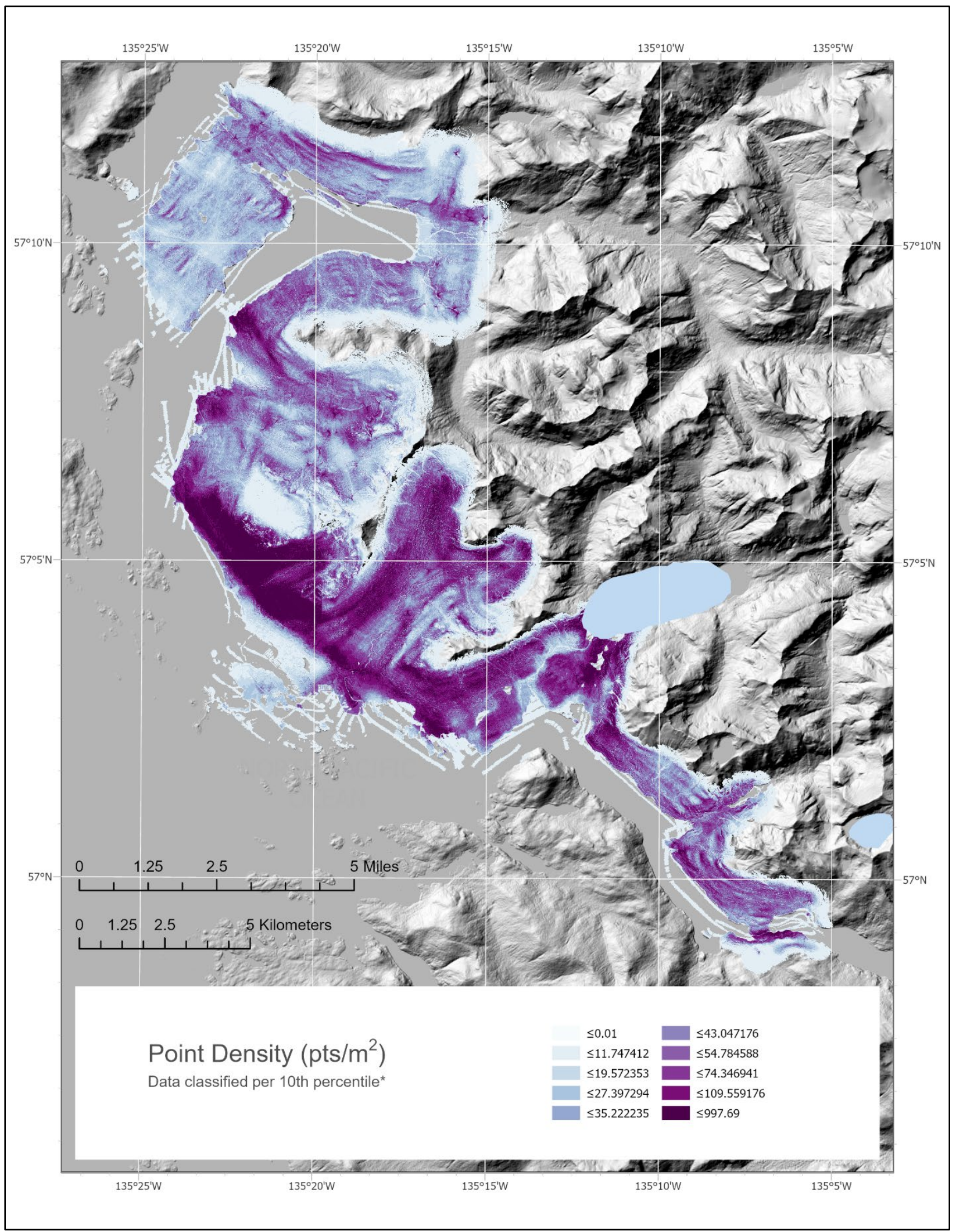

Figure 2. Ground point density displayed as 1-meter raster for the survey. 
Appendix 1. Control Points

\begin{tabular}{|c|c|c|c|c|c|}
\hline Number & Easting (m) & Northing (m) & $\begin{array}{l}\text { Known Z } \\
(\mathrm{m})\end{array}$ & $\begin{array}{l}\text { Laser Z } \\
(\mathrm{m})\end{array}$ & $\mathrm{Dz}(\mathrm{m})$ \\
\hline gсp-1 & 484645.2 & 6321154 & 26.221 & 26.24 & 0.019 \\
\hline gсp-3 & 484888.8 & 6321141 & 21.967 & 22.01 & 0.043 \\
\hline gсp-5 & 484957.2 & 6321237 & 15.923 & 15.97 & 0.047 \\
\hline gсp-7 & 485002.1 & 6321343 & 12.151 & 12.2 & 0.049 \\
\hline gсp-9 & 485111.2 & 6321425 & 10.418 & 10.43 & 0.012 \\
\hline gcp-11 & 485224.2 & 6321532 & 8.183 & 8.19 & 0.007 \\
\hline gcp-13 & 485323 & 6321655 & 8.646 & 8.7 & 0.054 \\
\hline gcp-15 & 485509.6 & 6321717 & 11.955 & 11.85 & -0.105 \\
\hline gcp-17 & 487755.5 & 6322435 & 10.901 & 10.93 & 0.029 \\
\hline gсp-19 & 487766.4 & 6322440 & 11.192 & 11.31 & 0.118 \\
\hline gcp-21 & 485931.6 & 6323021 & 14.301 & 14.34 & 0.039 \\
\hline gcp-23 & 485878.9 & 6322963 & 15.377 & 15.43 & 0.053 \\
\hline gсp-25 & 485849.9 & 6322873 & 14.096 & 14.01 & -0.086 \\
\hline gcp-27 & 485853.8 & 6322734 & 10.466 & 10.4 & -0.066 \\
\hline gсp-29 & 485509.6 & 6321717 & 11.924 & 11.85 & -0.074 \\
\hline gcp-31 & 483340.7 & 6321938 & 43.297 & 43.26 & -0.037 \\
\hline gсp-33 & 483294.9 & 6321980 & 44.145 & 44.14 & -0.005 \\
\hline gcp-35 & 481872.6 & 6322713 & 7.532 & 7.5 & -0.032 \\
\hline gсp-37 & 481328.7 & 6323880 & 22.854 & 22.86 & 0.006 \\
\hline gсp-39 & 481041.9 & 6324038 & 36.103 & 36.08 & -0.023 \\
\hline gсp-41 & 479915.6 & 6323625 & 12.02 & 12.02 & 0 \\
\hline gcp-43 & 478971.4 & 6324535 & 29.73 & 29.73 & 0 \\
\hline gсp-45 & 478967.7 & 6324501 & 29.339 & 29.35 & 0.011 \\
\hline gcp-47 & 478939.4 & 6324730 & 27.882 & 27.91 & 0.028 \\
\hline gср-49 & 478944.6 & 6324762 & 28.851 & 28.83 & -0.021 \\
\hline gcp-51 & 478120.8 & 6325871 & 59.968 & 59.93 & -0.038 \\
\hline gсp-53 & 476434.6 & 6328664 & 55.13 & 55.18 & 0.05 \\
\hline gcp-55 & 475833.8 & 6329480 & 9.747 & 9.73 & -0.017 \\
\hline gсp-59 & 478029.1 & 6322993 & 8.563 & 8.56 & -0.003 \\
\hline gcp-61 & 478087.2 & 6322977 & 7.739 & 7.73 & -0.009 \\
\hline gсp-63 & 476656 & 6327884 & 45.875 & 45.82 & -0.055 \\
\hline gcp-65 & 476842.5 & 6327692 & 42.003 & 41.94 & -0.063 \\
\hline gср-67 & 476809.7 & 6327688 & 40.814 & 40.85 & 0.036 \\
\hline gсp-69 & 476773.3 & 6327626 & 35.784 & 35.76 & -0.024 \\
\hline gсp-71 & 477596 & 6326152 & 52.179 & 52.2 & 0.021 \\
\hline gcp-73 & 477609.8 & 6326102 & 48.033 & 47.99 & -0.043 \\
\hline gcp-75 & 477614.2 & 6326040 & 42.007 & 41.99 & -0.017 \\
\hline gсp-77 & 477590.5 & 6326006 & 41.492 & 41.46 & -0.032 \\
\hline
\end{tabular}




\begin{tabular}{|c|c|c|c|c|c|}
\hline Number & Easting (m) & Northing $(\mathrm{m})$ & $\begin{array}{l}\text { Known Z } \\
(\mathrm{m})\end{array}$ & $\begin{array}{l}\text { Laser Z } \\
(\mathrm{m})\end{array}$ & Dz $(\mathrm{m})$ \\
\hline gcp-79 & 477648.3 & 6325977 & 37.94 & 37.97 & 0.03 \\
\hline gcp-81 & 479063 & 6323738 & 16.498 & 16.57 & 0.072 \\
\hline gcp-83 & 480627.9 & 6322920 & 10.453 & 10.39 & -0.063 \\
\hline gcp-85 & 480626.5 & 6322898 & 9.528 & 9.52 & -0.008 \\
\hline gcp-87 & 480824.3 & 6323839 & 28.724 & 28.73 & 0.006 \\
\hline gcp-89 & 480805.7 & 6323839 & 28.701 & 28.73 & 0.029 \\
\hline gсp-91 & 480815.4 & 6323826 & 29.065 & 29.02 & -0.045 \\
\hline gcp-93 & 481891.8 & 6323208 & 65.539 & 65.5 & -0.039 \\
\hline gcp-95 & 482001.1 & 6323072 & 60.137 & 60.16 & 0.023 \\
\hline gcp-97 & 482034.1 & 6323066 & 61.05 & 60.93 & -0.12 \\
\hline gсp-101 & 486767.4 & 6324291 & 80.421 & 80.33 & -0.091 \\
\hline gcp-103 & 487793 & 6324321 & 142.228 & 142.28 & 0.052 \\
\hline gcp-105 & 487788.1 & 6324340 & 142.354 & 142.29 & -0.064 \\
\hline gсp-107 & 487769.8 & 6324361 & 144.967 & 144.92 & -0.047 \\
\hline gсp-109 & 487787.9 & 6321603 & 17.551 & 17.56 & 0.009 \\
\hline gсp-111 & 491001.7 & 6319067 & 8.935 & 8.93 & -0.005 \\
\hline gcp-113 & 490976.8 & 6319101 & 6.743 & 6.73 & -0.013 \\
\hline gсp-115 & 493053 & 6315957 & 124.214 & 124.2 & -0.014 \\
\hline gcp-117 & 493064.1 & 6315995 & 123.736 & 123.64 & -0.096 \\
\hline gсp-119 & 485709.7 & 6323162 & 56.125 & 56.02 & -0.105 \\
\hline gсp-121 & 485603.4 & 6323105 & 103.947 & 103.96 & 0.013 \\
\hline gсp-123 & 485442.1 & 6323071 & 105.617 & 105.53 & -0.087 \\
\hline gcp-125 & 485204 & 6323003 & 119.852 & 119.73 & -0.122 \\
\hline gсp-127 & 485081.3 & 6322897 & 112.44 & 112.44 & 0 \\
\hline gcp-131 & 484815.5 & 6322649 & 59.398 & 59.38 & -0.018 \\
\hline gсp-133 & 484660 & 6322461 & 49.51 & 49.38 & -0.13 \\
\hline gсp-135 & 484574.9 & 6322294 & 39.875 & 39.72 & -0.155 \\
\hline gсp-137 & 484498.7 & 6322103 & 39.256 & 39.19 & -0.066 \\
\hline gcp-139 & 484506.4 & 6321839 & 39.19 & 39.01 & -0.18 \\
\hline gсp-141 & 486048.3 & 6322513 & 6.696 & 6.71 & 0.014 \\
\hline gсp-143 & 486069.7 & 6322635 & 6.776 & 6.72 & -0.056 \\
\hline gсp-145 & 486053.2 & 6322644 & 7.058 & 7.03 & -0.028 \\
\hline gcp-147 & 486076.1 & 6322619 & 6.63 & 6.66 & 0.03 \\
\hline gср-149 & 486045 & 6322803 & 7.442 & 7.4 & -0.042 \\
\hline gcp-151 & 477848.5 & 6332278 & 11.967 & 11.96 & -0.007 \\
\hline gсp-153 & 477807.9 & 6332249 & 11.829 & 11.83 & 0.001 \\
\hline gcp-155 & 477764.3 & 6332527 & 9.175 & 9.14 & -0.035 \\
\hline gcp-157 & 479078.5 & 6331430 & 18.195 & 18.21 & 0.015 \\
\hline gсp-159 & 479098.3 & 6331411 & 17.989 & 17.98 & -0.009 \\
\hline gcp-161 & 479376.8 & 6331555 & 25.316 & 25.36 & 0.044 \\
\hline
\end{tabular}




\begin{tabular}{|c|c|c|c|c|c|}
\hline Number & Easting (m) & Northing (m) & \begin{tabular}{|l} 
Known Z \\
$(\mathrm{m})$
\end{tabular} & \begin{tabular}{|l}
$\begin{array}{l}\text { Laser Z } \\
(\mathrm{m})\end{array}$ \\
\end{tabular} & $\mathrm{Dz}(\mathrm{m})$ \\
\hline gсp-163 & 480093 & 6330753 & 71.753 & 71.8 & 0.047 \\
\hline gсp-169 & 480565.5 & 6331205 & 51.427 & 51.36 & -0.067 \\
\hline gсp-171 & 480692.9 & 6331170 & 53.62 & 53.55 & -0.07 \\
\hline gсp-173 & 480807.3 & 6331133 & 57.193 & 57.14 & -0.053 \\
\hline gсp-177 & 481075.3 & 6331562 & 263.528 & 263.65 & 0.122 \\
\hline gcp-183 & 477291.9 & 6331895 & 5.423 & 5.35 & -0.073 \\
\hline gсp-185 & 476919.5 & 6339310 & 3.636 & 3.78 & 0.144 \\
\hline gcp-187 & 477003.9 & 6339322 & 3.871 & 3.9 & 0.029 \\
\hline gсp-189 & 476114.3 & 6338670 & 4.096 & 4.08 & -0.016 \\
\hline gcp-195 & 476119.9 & 6333387 & 4.228 & 4.05 & -0.178 \\
\hline gсp-197 & 477088.3 & 6335280 & 4.44 & 4.37 & -0.07 \\
\hline gcp-203 & 479059.1 & 6336535 & 4.317 & 4.32 & 0.003 \\
\hline gcp-207 & 478971.1 & 6337872 & 3.847 & 3.89 & 0.043 \\
\hline gсp-209 & 480073.7 & 6337653 & 3.763 & 3.79 & 0.027 \\
\hline gсp-211 & 479906.5 & 6337470 & 3.919 & 3.86 & -0.059 \\
\hline gcp-213 & 481832 & 6337165 & 3.802 & 3.92 & 0.118 \\
\hline gcp-215 & 481868.1 & 6337172 & 3.863 & 3.75 & -0.113 \\
\hline gcp-217 & 482853.8 & 6336843 & 3.802 & 3.88 & 0.078 \\
\hline gcp-221 & 482626.4 & 6335495 & 5.17 & 5.14 & -0.03 \\
\hline gcp-223 & 482665.9 & 6335500 & 3.713 & 3.77 & 0.057 \\
\hline gcp-227 & 481355.2 & 6335655 & 3.364 & 3.38 & 0.016 \\
\hline gcp-231 & 479789.1 & 6335529 & 3.723 & 3.78 & 0.057 \\
\hline gcp-233 & 477291.9 & 6331895 & 5.421 & 5.35 & -0.071 \\
\hline
\end{tabular}

\begin{tabular}{||l||r|}
\hline Average dz $(\mathrm{m})$ & -0.015 \\
\hline \hline Minimum dz $(\mathrm{m})$ & -0.18 \\
\hline \hline Maximum dz $(\mathrm{m})$ & 0.144 \\
\hline \hline Average magnitude $(\mathrm{m})$ & 0.048 \\
\hline \hline RMSE $(\mathrm{m})$ & $\mathbf{0 . 0 6 3}$ \\
\hline \hline Standard deviation $(\mathrm{m})$ & $\mathbf{0 . 0 6 2}$ \\
\hline
\end{tabular}


Appendix 2. Checkpoints

\begin{tabular}{|c|c|c|c|c|c|}
\hline Number & Easting (m) & Northing (m) & $\begin{array}{l}\text { Known Z } \\
(\mathrm{m})\end{array}$ & $\begin{array}{l}\text { Laser Z } \\
(\mathrm{m})\end{array}$ & $\mathrm{Dz}(\mathrm{m})$ \\
\hline gсp-2 & 484863 & 6321108 & 23.335 & 23.3 & -0.035 \\
\hline gсp-4 & 484910.3 & 6321179 & 20.031 & 20.04 & 0.009 \\
\hline gсp-6 & 484997.7 & 6321296 & 13.478 & 13.56 & 0.082 \\
\hline gсp-8 & 485025.2 & 6321382 & 11.078 & 11.1 & 0.022 \\
\hline gcp-10 & 485165.8 & 6321475 & 9.356 & 9.35 & -0.006 \\
\hline gcp-12 & 485311.8 & 6321601 & 8.784 & 8.86 & 0.076 \\
\hline gcp-14 & 485413.8 & 6321701 & 10.224 & 10.21 & -0.014 \\
\hline gcp-16 & 487759.3 & 6322427 & 10.842 & 10.91 & 0.068 \\
\hline gcp-18 & 487755.6 & 6322448 & 10.865 & 10.88 & 0.015 \\
\hline gсp-20 & 487774 & 6322421 & 11.225 & 11.27 & 0.045 \\
\hline gcp-22 & 485904.4 & 6322997 & 15.095 & 15.15 & 0.055 \\
\hline gcp-24 & 485859.6 & 6322921 & 14.993 & 15 & 0.007 \\
\hline gcp-26 & 485847.8 & 6322808 & 12.784 & 12.75 & -0.034 \\
\hline gcp-28 & 485870 & 6322676 & 9.043 & 9.07 & 0.027 \\
\hline gсp-30 & 485509.6 & 6321717 & 11.923 & 11.85 & -0.073 \\
\hline gcp-32 & 483315.1 & 6321957 & 44.156 & 44.14 & -0.016 \\
\hline gсp-34 & 481878.1 & 6322696 & 7.037 & 7.06 & 0.023 \\
\hline gcp-36 & 481524.1 & 6323292 & 27.432 & 27.42 & -0.012 \\
\hline gсp-38 & 481288.5 & 6323913 & 23.063 & 23.02 & -0.043 \\
\hline gcp-40 & 481036.3 & 6324139 & 37.361 & 37.33 & -0.031 \\
\hline gср-42 & 479818.4 & 6324459 & 36.283 & 36.28 & -0.003 \\
\hline gcp-44 & 479002.1 & 6324524 & 29.369 & 29.4 & 0.031 \\
\hline gсp-46 & 478921.3 & 6324747 & 28.137 & 28.12 & -0.017 \\
\hline gcp-48 & 478968.1 & 6324752 & 28.815 & 28.81 & -0.005 \\
\hline gсp-50 & 478027 & 6325429 & 45.473 & 45.56 & 0.087 \\
\hline gcp-52 & 476900.3 & 6326944 & 9.867 & 9.84 & -0.027 \\
\hline gсp-54 & 476440.5 & 6328660 & 55.191 & 55.25 & 0.059 \\
\hline gcp-60 & 478029.1 & 6322993 & 8.561 & 8.56 & -0.001 \\
\hline gсp-62 & 477529.9 & 6329109 & 143.77 & 143.88 & 0.11 \\
\hline gcp-64 & 476814 & 6327701 & 41.635 & 41.68 & 0.045 \\
\hline gсp-66 & 476852.3 & 6327679 & 41.79 & 41.74 & -0.05 \\
\hline gcp-68 & 476794.7 & 6327660 & 38.543 & 38.57 & 0.027 \\
\hline gср-70 & 476755.7 & 6327595 & 33.153 & 33.09 & -0.063 \\
\hline gсp-72 & 477603.2 & 6326129 & 50.547 & 50.53 & -0.017 \\
\hline gсp-74 & 477613.4 & 6326068 & 44.585 & 44.52 & -0.065 \\
\hline gсp-76 & 477590.6 & 6326030 & 41.729 & 41.69 & -0.039 \\
\hline gсp-78 & 477615.4 & 6326003 & 38.477 & 38.36 & -0.117 \\
\hline gcp-80 & 477717.4 & 6325911 & 40.798 & 40.83 & 0.032 \\
\hline
\end{tabular}




\begin{tabular}{|c|c|c|c|c|c|}
\hline Number & Easting (m) & Northing (m) & $\begin{array}{l}\text { Known Z } \\
\text { (m) }\end{array}$ & $\begin{array}{l}\text { Laser Z } \\
(\mathrm{m})\end{array}$ & Dz (m) \\
\hline gсp-82 & 479077 & 6323743 & 17.425 & 17.54 & 0.115 \\
\hline gсp-84 & 480615 & 6322905 & 9.897 & 9.87 & -0.027 \\
\hline gcp-86 & 480626.6 & 6322937 & 11.208 & 11.19 & -0.018 \\
\hline gсp-88 & 480817.2 & 6323849 & 28.582 & 28.6 & 0.018 \\
\hline gсp-90 & 480815.4 & 6323826 & 29.061 & 29.02 & -0.041 \\
\hline gсp-92 & 481775.4 & 6323103 & 37.358 & 37.33 & -0.028 \\
\hline gсp-94 & 481879 & 6323190 & 64.166 & 64.14 & -0.026 \\
\hline gсp-96 & 482017.4 & 6323054 & 61.005 & 61.04 & 0.035 \\
\hline gсp-100 & 485499.2 & 6321719 & 12.136 & 12.21 & 0.074 \\
\hline gcp-102 & 487813.1 & 6324333 & 142.422 & 142.46 & 0.038 \\
\hline gcp-104 & 487773.8 & 6324329 & 142.376 & 142.36 & -0.016 \\
\hline gcp-106 & 487757.3 & 6324334 & 142.519 & 142.41 & -0.109 \\
\hline gсp-108 & 486475.3 & 6322680 & 23.679 & 23.74 & 0.061 \\
\hline gcp-110 & 489427.3 & 6320005 & 14.497 & 14.49 & -0.007 \\
\hline gсp-112 & 490991.5 & 6319117 & 8.451 & 8.46 & 0.009 \\
\hline gcp-114 & 491721.6 & 6316573 & 36.249 & 36.25 & 0.001 \\
\hline gcp-116 & 493068.6 & 6315969 & 123.776 & 123.61 & -0.166 \\
\hline gcp-118 & 485709.7 & 6323162 & 56.115 & 56.02 & -0.095 \\
\hline gcp-120 & 485622.3 & 6323261 & 86.334 & 86.32 & -0.014 \\
\hline gcp-122 & 485538.7 & 6323058 & 104.083 & 103.88 & -0.203 \\
\hline gcp-124 & 485287.9 & 6323020 & 105.698 & 105.63 & -0.068 \\
\hline gcp-126 & 485151.4 & 6322951 & 119.026 & 119 & -0.026 \\
\hline gсp-130 & 484878 & 6322752 & 65.464 & 65.49 & 0.026 \\
\hline gcp-134 & 484607.3 & 6322360 & 43.87 & 43.83 & -0.04 \\
\hline gсp-138 & 484508.9 & 6321933 & 37.263 & 37.27 & 0.007 \\
\hline gcp-142 & 486052.7 & 6322538 & 6.924 & 6.92 & -0.004 \\
\hline gсp-144 & 486061.6 & 6322648 & 6.855 & 6.83 & -0.025 \\
\hline gcp-146 & 486052.7 & 6322621 & 7.273 & 7.26 & -0.013 \\
\hline gсp-148 & 486047.2 & 6322792 & 7.339 & 7.31 & -0.029 \\
\hline gсp-150 & 486042.2 & 6322817 & 7.475 & 7.46 & -0.015 \\
\hline gcp-152 & 477829.9 & 6332265 & 11.832 & 11.85 & 0.018 \\
\hline gсp-154 & 477754.2 & 6332515 & 8.669 & 8.62 & -0.049 \\
\hline gcp-156 & 478145.6 & 6332014 & 9.105 & 9.17 & 0.065 \\
\hline gсp-158 & 479105.9 & 6331451 & 18.392 & 18.31 & -0.082 \\
\hline gсp-160 & 479073.9 & 6331578 & 20.587 & 20.59 & 0.003 \\
\hline gсp-166 & 480422 & 6330203 & 78.07 & 78.2 & 0.13 \\
\hline gcp-168 & 480548.5 & 6331188 & 51.027 & 51.02 & -0.007 \\
\hline gсp-170 & 480603.8 & 6331206 & 52.015 & 52.04 & 0.025 \\
\hline gcp-172 & 480776.1 & 6331158 & 56.127 & 56.26 & 0.133 \\
\hline gср-174 & 481070.8 & 6331218 & 89.83 & 89.87 & 0.04 \\
\hline
\end{tabular}




\begin{tabular}{|c|c|c|c|c|c|}
\hline Number & Easting (m) & Northing (m) & \begin{tabular}{|l} 
Known Z \\
$(\mathrm{m})$
\end{tabular} & \begin{tabular}{|l}
$\begin{array}{l}\text { Laser Z } \\
(\mathrm{m})\end{array}$ \\
\end{tabular} & $\mathrm{Dz}(\mathrm{m})$ \\
\hline gсp-176 & 481082.3 & 6331557 & 263.634 & 263.41 & -0.224 \\
\hline gcp-184 & 476876.9 & 6339287 & 3.846 & 4 & 0.154 \\
\hline gсp-186 & 476949.9 & 6339336 & 3.428 & 3.52 & 0.092 \\
\hline gсp-188 & 476106.9 & 6338671 & 4.059 & 3.84 & -0.219 \\
\hline gсp-190 & 476077.2 & 6338610 & 3.798 & 3.95 & 0.152 \\
\hline gcp-200 & 478073.4 & 6335814 & 4.286 & 4.38 & 0.094 \\
\hline gсp-204 & 478834.7 & 6337836 & 4.178 & 4.34 & 0.162 \\
\hline gсp-206 & 478936.6 & 6337839 & 3.871 & 4 & 0.129 \\
\hline gсp-208 & 480060.5 & 6337667 & 3.639 & 3.81 & 0.171 \\
\hline gcp-212 & 479900.2 & 6337474 & 4.263 & 4.1 & -0.163 \\
\hline gсp-214 & 481850.4 & 6337164 & 3.869 & 3.87 & 0.001 \\
\hline gcp-216 & 482832.1 & 6336875 & 3.64 & 3.85 & 0.21 \\
\hline gcp-222 & 482626.5 & 6335495 & 5.18 & 5.15 & -0.03 \\
\hline gcp-224 & 482666 & 6335499 & 3.702 & 3.82 & 0.118 \\
\hline gср-226 & 481335.8 & 6335664 & 3.805 & 3.93 & 0.125 \\
\hline gcp-228 & 481328.4 & 6335653 & 4.744 & 4.82 & 0.076 \\
\hline gсp-230 & 479799.8 & 6335593 & 3.47 & 3.5 & 0.03 \\
\hline gcp-234 & 477291.9 & 6331895 & 5.429 & 5.35 & -0.079 \\
\hline
\end{tabular}

\begin{tabular}{||l|r|}
\hline \hline Average dz $(\mathrm{m})$ & $\mathbf{0 . 0 0 7}$ \\
\hline \hline Minimum dz $(\mathrm{m})$ & -0.224 \\
\hline \hline Maximum dz $(\mathrm{m})$ & $\mathbf{0 . 2 1}$ \\
\hline \hline Average magnitude $(\mathrm{m})$ & $\mathbf{0 . 0 5 9}$ \\
\hline \hline RMSE $(\mathrm{m})$ & $\mathbf{0 . 0 8}$ \\
\hline \hline Standard deviation $(\mathrm{m})$ & $\mathbf{0 . 0 8}$ \\
\hline
\end{tabular}

